Research Article

\title{
Two Years after Loading Performance of Implant-Supported Overdenture with Metal Bar and Low-Profile Attachments: A Prospective Case Series Multicenter Clinical Study
}

\author{
Marco Montanari, ${ }^{1}$ Marco Tallarico $\mathbb{D D}^{1,2,3}$ Gabriele Vaccaro, ${ }^{1}$ Emiliano Ferrari, ${ }^{1}$ \\ Roberto Scrascia, ${ }^{1}$ Luca Ortensi, ${ }^{1}$ Marco Cicciù, ${ }^{4}$ Silvio Mario Meloni, ${ }^{1,2,3}$ \\ Aurea Immacolata Lumbau, ${ }^{3}$ Marianna Avrampou, ${ }^{5}$ and Matteo Martinollii \\ ${ }^{1}$ Private Practice, Rome, Italy \\ ${ }^{2}$ Implantology and Prosthetic Aspects, Master of Science in Dentistry Program, Aldent University, Tirana, Albania \\ ${ }^{3}$ Surgical, Micro-surgical and Medical Science Department, University of Sassari, Sassari, Italy \\ ${ }^{4}$ Department of Biomedical and Dental Sciences Morphological and Functional Images, School of Dentistry, \\ University of Messina, Messina, Italy \\ ${ }^{5}$ Department of Prosthodontics, Tufts University School of Dental Medicine, Boston, USA
}

Correspondence should be addressed to Marco Tallarico; me@studiomarcotallarico.it

Received 14 July 2020; Revised 30 August 2020; Accepted 6 September 2020; Published 18 September 2020

Academic Editor: Gaetano Isola

Copyright (c) 2020 Marco Montanari et al. This is an open access article distributed under the Creative Commons Attribution License, which permits unrestricted use, distribution, and reproduction in any medium, provided the original work is properly cited.

\begin{abstract}
Background. Prosthetic rehabilitation of patients with atrophic arches can be very challenging. Purpose. The aim of the present prospective multicenter study was to report the two-year preliminary data on clinical and radiographic performance of implantsupported overdentures with a metal bar and low-profile attachments. Material and Methods. A computer-aided design/ computer-aided manufacturing (CAD/CAM) titanium bar or a conventional cast metal bar was fabricated according to the anatomy of the ridge, prosthetic contours (teeth setup), and implant position. Three to six threadable OT Equator attachments (Rhein 83, Bologna, Italy) were placed along the implant bar. A cobalt-chromium alloy metal framework was fabricated and fitted onto the metal bar as a counterpart. Prosthetic survival rate, biologic and technical complications, peri-implant bone loss, changes in oral health impact profile index, bleeding on probing, and plaque index were reported. Results. Overall, 177 implants were placed (range three to six) to support 43 metal bars with 170 OT Equators (Rhein 83, range three to six). Eleven metal bars were fabricated using CAD/CAM technology, while the other 32 were conventionally produced using cast technique. All the participants were followed up for at least two years (mean 42.2 months, range $24-88$ months) after prosthesis delivery. Two maxillary implants failed in one nonsmoking patient (1.1\%). The 2-year prosthesis survival rate was $97.7 \%$. Only three minor technical complications were reported. Two years after loading, the mean marginal bone loss was $0.22 \pm 0.09 \mathrm{~mm}$ (95\% CI: 0.16 to 0.26 ). Two years after loading, OHIP was $22.3 \pm 7.1$ (95\% CI from 17.4 to 24.6). Compared to the baseline, the difference was statistically significant $(P \leq 0.001)$. At the two-year follow-up session, successful periodontal parameters were experienced. Conclusions. Implant overdenture supported by a CAD/CAM titanium bar may be a reliable option for the treatment of the edentulous arch over a 2-year period. Oral health-related quality of life significantly improved in all treated participants.
\end{abstract}

\section{Introduction}

Periodontitis involves a severe chronic inflammation that can lead to gradual teeth loss. Moreover, accumulating evidence suggests that periodontitis is associated with increased risk for cardiovascular diseases $[1,2]$. Chemotherapeutic agents have been widely used as adjuncts for the treatment of chronic periodontitis [3]. Dental implants have become widely used to replace missing teeth for patients suffering from tooth loss. Because inflammatory periodontal 
disease is a ubiquitous and irreversible inflammatory condition and represents one of the main reasons for tooth loss, the issue in regard to successful implant placement becomes a major consideration for the clinicians.

Many treatment options exist for the treatment of completely edentulous patients, ranging from conventional complete dentures to fixed implant-supported restorations. Establishing the most adequate treatment option for the edentulous patients still remains a challenging task. The use of osseointegrated implants for the retention of an overdenture has a favorable impact on the patient's quality of life [4], improving the masticatory performance when compared to the conventional removable denture [5]. Nevertheless, biological and technical complications occur continuously over time as a result of fatigue and stress $[6,7]$. Accurate diagnosis and evidence-based treatment planning are critically important to reduce complications and obtain longterm predictable outcomes.

Several factors play a major role in the treatment planning of the edentulous patient, including but not limiting to anatomy, biomechanics (i.e., available interocclusal space, occlusion, and neuromuscular function), esthetics, phonetics, cost, amount of repair and maintenance needed, patient compliance, and expectations. Since the patient and dentist preferences influence the type of prosthesis delivered, it is likely that knowledge of indications and contraindications, including benefits and limitations of implant-related treatment options, is mandatory $[8,9]$.

Detailed diagnostic examination in combination with patients' needs and expectations should be the basis to choose the appropriate type of restoration before the surgical procedure has been started [8]. Newer technologies such as computer-assisted template-based implant placement may play an important role in achieving predictable results from an esthetic and functional point of view [9]. Implant-supported prostheses can be either fixed or removable. Each option offers different levels of function and comfort with its own indications and contraindications. Patients with moderate to advanced alveolar ridge resorption can be rehabilitated with either type of prosthesis. Nevertheless, the presence of adequate lip support is one of the most important criteria in the selection of a fixed versus removable implant-based restoration $[10,11]$. Removable implant prostheses included either implant-retained or implant-supported options. An implant-retained overdenture with single attachments requires a minimum of two implants in the mandible and four implants in the maxilla. The masticatory forces are transferred to the alveolar mucosa and consequently the underlying bone, while the dental implants provide retention to the denture. An implant-supported overdenture with a bar requires a minimum of four implants in both mandible and maxilla [9]. The masticatory forces are transferred completely to the dental implants, increasing patients' comfort, but it can be removed by the patient to maintain proper oral hygiene. Implant overdentures are the treatment of choice in cases requiring extensive lip support since this type of prosthesis can provide better esthetic results with an extended buccal flange.

Completely edentulous patients often present with moderate to high bone resorption. Avrampou and coworkers [9] demonstrated that in the majority of their cohort of patients, the available space between the prosthetic crown and the implant platform had to be compensated with the prosthetic material, making a buccal flange needed to provide lip and facial support. For the latter, the need for perioral soft tissue support with a prosthetic flange should always be considered even if implants can be placed in a proper position [8-11]. Moreover, a removable implantsupported prosthesis may be a feasible option to overcome the technical complications of other treatment options [10-12]. Finally, this type of implant-supported restoration gives high patient's satisfaction with the advantage of retrievability just like screw-retained prosthesis, making oral hygiene and maintenance easier.

The aim of the present multicenter prospective clinical study was to report clinical and radiographic data on patients rehabilitated with an implant-supported overdentures retained by a titanium computer-aided design/computeraided manufacturing (CAD/CAM) bar and low-profile attachments. The study was written according to the STROBE (Strengthening the Reporting of Observational Studies in Epidemiology) guidelines [13].

\section{Materials and Methods}

This study was designed as a multicenter prospective case series study conducted according to the Declaration of Helsinki of 1975, as revised in 2008. The publication of the data was approved by the Institutional Review Board of the Aldent University, Tirana, Albania (4/2020).

Any edentulous patient in at least one arch, aged 18 years or older, requiring an implant overdenture to rehabilitate a complete edentulous arch was considered eligible for this study and treated in consecutive order after being informed about the nature of the study and providing a written informed consent. If patients were edentulous on both arches, only one bar was considered. Additional inclusion criteria were Cawood and Howell class III to VI [11], patients refusing guided bone reconstruction, and the need for lip support. Exclusion criteria were general contraindications to oral surgery, pregnancy or nursing, intravenous bisphosphonate therapy, alcohol or drug abuse, heavy smoking $(\geq 10$ cigarettes/day), immediate postextractive implants, irradiation therapy to the head or neck region within the last five years, untreated periodontitis, full-mouth bleeding on probing and a full-mouth plaque index of $\leq 25 \%$, absence of teeth in the opposite arch, and allergy or adverse reactions to the restorative materials (Table 1).

Patients were consecutively enrolled and treated in five centers in Italy between January 2011 and March 2016. The study protocol was designed to collect data up to two years after implant loading. This manuscript presents the 
TABLE 1: Inclusion and exclusion criteria.

\begin{tabular}{|c|c|}
\hline Inclusion criteria & Exclusion criteria \\
\hline $\begin{array}{l}\text { Aged } 18 \text { years or older } \\
\text { Signed informed consent } \\
\text { Need of an implant overdenture in one arch } \\
\text { Cawood and Howell class III to VI } \\
\text { Two years after loading follow-up } \\
\text { Available radiographs (implant loading and follow-ups) } \\
\text { Patients refusing guided bone reconstruction } \\
\text { Need for lip support }\end{array}$ & $\begin{array}{c}\text { General contraindications to oral surgery } \\
\text { Pregnancy or nursing } \\
\text { Treatment with intravenous bisphosphonates } \\
\text { Substance abuse (drugs or alcohol) } \\
\text { Heavy smokers }(\geq 11 \text { cigarettes per day) } \\
\text { Immediate postextractive implants } \\
\text { Irradiation of the neck or head area in the past } 5 \text { years } \\
\text { Poor oral hygiene (bleeding on probing }>25 \%) \\
\text { Untreated periodontitis } \\
\text { Absence of teeth in the opposite arch } \\
\text { Allergy or adverse reactions to the restorative materials } \\
\text { Less than two-year follow-up }\end{array}$ \\
\hline
\end{tabular}

preliminary data at a minimum of two years after loading examination.

\section{Surgical and Prosthetic Protocols}

Preoperative photographs, radiographs, study models, and facebow registration were produced for initial screening and case evaluation (Figure 1). The preexistent removable complete denture, if judged viable from an esthetic and functional perspective, was used to evaluate the maxillomandibular relationship and the amount of mucosal coverage; otherwise, a new complete removable denture was made according to the functional and esthetic requirements. A cone beam computed tomography (CBCT) scan (CRANEX 3Dx, SOREDEX, Tuusula, Finland) was taken to evaluate the bone quantity according to the Cawood and Howell classification [14].

One hour before implant placement, patients received a single dose of an antibiotic ( $2 \mathrm{~g}$ of amoxicillin or $600 \mathrm{mg}$ of clindamycin if allergic to penicillin) and professional hygiene therapy to the remain teeth of the opposing arch. Local anesthesia was administered and a mucoperiosteal flap was elevated. Three to six submerged implants were placed according to the manufacturer's guidelines. Clinician was free to place implants using a computer-assisted templatebased approach or freehand. In the mandible, the implants were placed in the interforaminal region, while in the maxilla, the implants were placed mesially to the sinus floor.

After surgery, patients received medication and oral hygiene instructions. A cold and soft diet was recommended for ten days. Smokers were encouraged to stop smoking for three days postoperatively.

Four months after the implant placement, definitive impressions were taken using a plaster (Snow White Plaster No. 2, Kerr, Orange, Calif., USA) or polyether material (Impregum, 3M ESPE, Seefeld, Germany) with a custom open tray (Diatray Top, Dental Kontor, Stockelsdorf, Germany). Models were mounted in a dental articulator in centric relation, using a facebow, at the established occlusal vertical dimension. Then, esthetics and function (phonetics and occlusion) were verified and approved by both the clinician and the patient at the try-in appointments. Afterward, a CAD/CAM or a conventional cast metal bar was fabricated according to the ridge and prosthetic contours and implant position. Four to five threadable OT Equator attachments (Rhein 83, Bologna, Italy) were placed along the implant bar. The accuracy of the implant bar was clinically and radiographically tested in the patient's mouth according to a previously published protocol [15]. A cobalt-chromium alloy metal framework was fabricated and fitted onto the metal bar as a counterpart. Finally, the overdenture was finished, using acrylic or composite teeth, and the borders were sealed to minimize food impaction and saliva and air leakage. The metal bar was screwed according to the manufacturer's instructions, and the final prostheses were delivered (Figures 2 and 3). Patients were recalled for hygiene maintenance every 4 months for the entire study period (Figure 4). Retentive caps were evaluated at each appointment and replaced if needed. Occlusion was checked at every appointment. Occlusion with anterior guidance was the first choice when antagonist was natural dentition or fixed prosthesis. Group function of the working side was chosen whenever the arch relationship did not allow the anterior guidance. Balanced occlusion was chosen when the antagonist was a complete removable denture.

3.1. Outcome Measures. The primary outcome measures were as follows:

Implants and prosthesis survival rates and failures: an implant was considered a failure if it presented with any mobility, progressive marginal bone loss, and suppuration or any mechanical complications rendering the implant unusable (i.e., implant fracture). A prosthesis was considered a failure if it needed to be replaced with another prosthesis for any reason.

Complications: any biological (pain, swelling, suppuration, etc.) and/or mechanical (screw loosening, fracture of the framework and/or the veneering material, etc.) complications were evaluated. Implants and prosthesis failures and complications were assessed and treated by the treating clinicians at each center.

Marginal bone loss (MBL): digital periapical radiographs were made with the paralleling technique using commercially available film holders. Mesial and distal bone level changes were measured as the distance from the implant shoulder and the most coronal bone to 


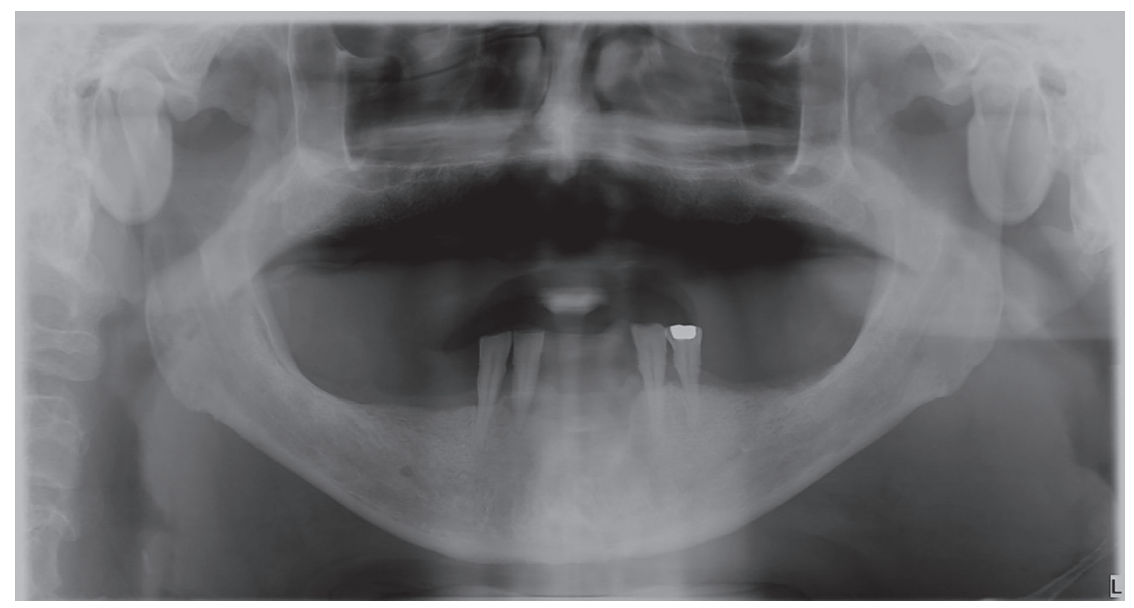

FIGURE 1: Preoperative panoramic radiograph.

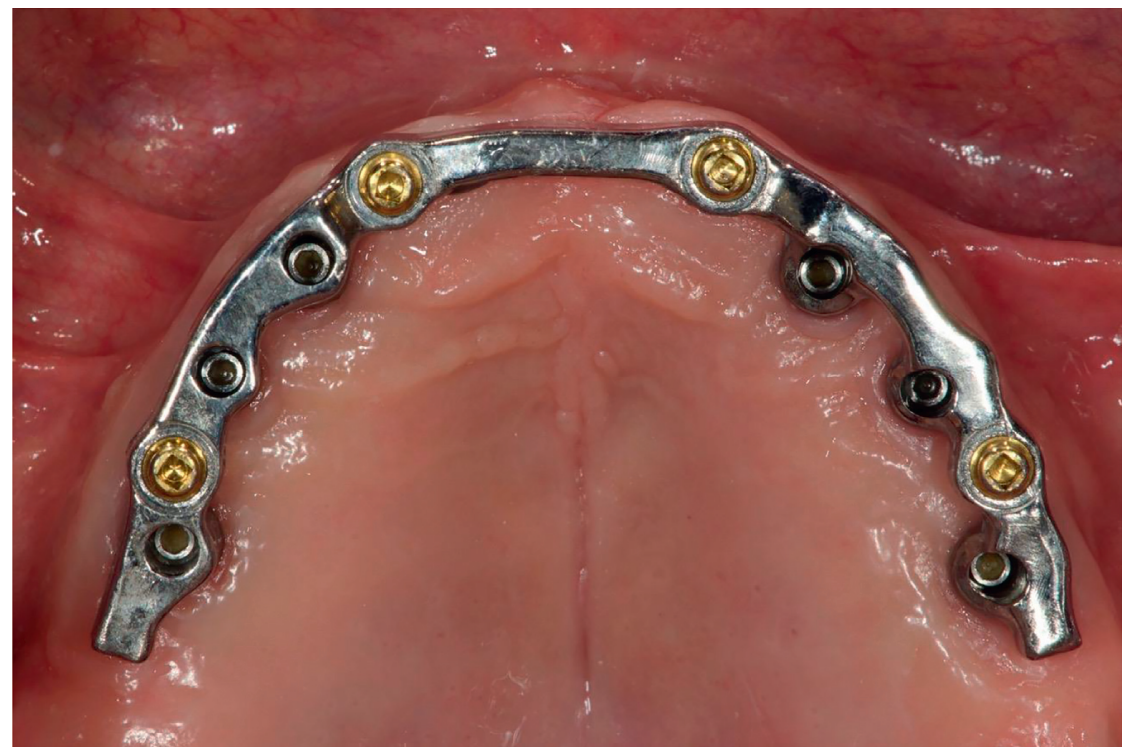

FIGURE 2: Intraoral clinical image of the CAD/CAM titanium bar with low-profile attachments (OT Equator attachments, Rhein 83, Bologna, Italy).

implant contact and then averaged. Radiographs were taken at the definitive prosthesis delivery (implant loading) and then every year. The difference between each follow-up and baseline was considered as marginal bone loss. An independent outcome assessor measured all the radiographs using a calibrated software (DFW2.8 for Windows, SOREDEX, Tuusula, Finland).

Oral Health Impact Profile (OHIP-21): a questionnaire with 21 questions, divided into seven subscales (functional limitations, physical pain, psychological discomfort, physical disability, psychological disability, social disability, and handicap), with two to four questions each, was completed by patients. Patients were instructed to choose from five possible responses ranging from 1 (never) to 5 (very often). The questionnaire was administered by an independent dentist before treatment and yearly after definitive prosthesis delivery.
Bleeding index and plaque index were evaluated yearly around each implant-abutment interface using a periodontal probe (PCPUNC156, Hu-Friedy, Milan, Italy) by an independent blinded dental hygienist. Four sites were evaluated (yes $=1 /$ no $=0$ ) at each implantabutment complex and averaged between them.

\section{Statistical Analysis}

Because there are only few reports in the literature that studied this topic, a sample size calculation was not calculated. All data analysis was carried out according to a preestablished analysis plan using SPSS Statistics for Macintosh (version 22.0, IBM, Armonk, NY, USA). Descriptive analysis was performed using means, standard deviations, and a 95\% confidence interval, as well as median and interquartile ranges (IQR: first quartile; median; third quartile). The Fisher exact test for count data was used to 


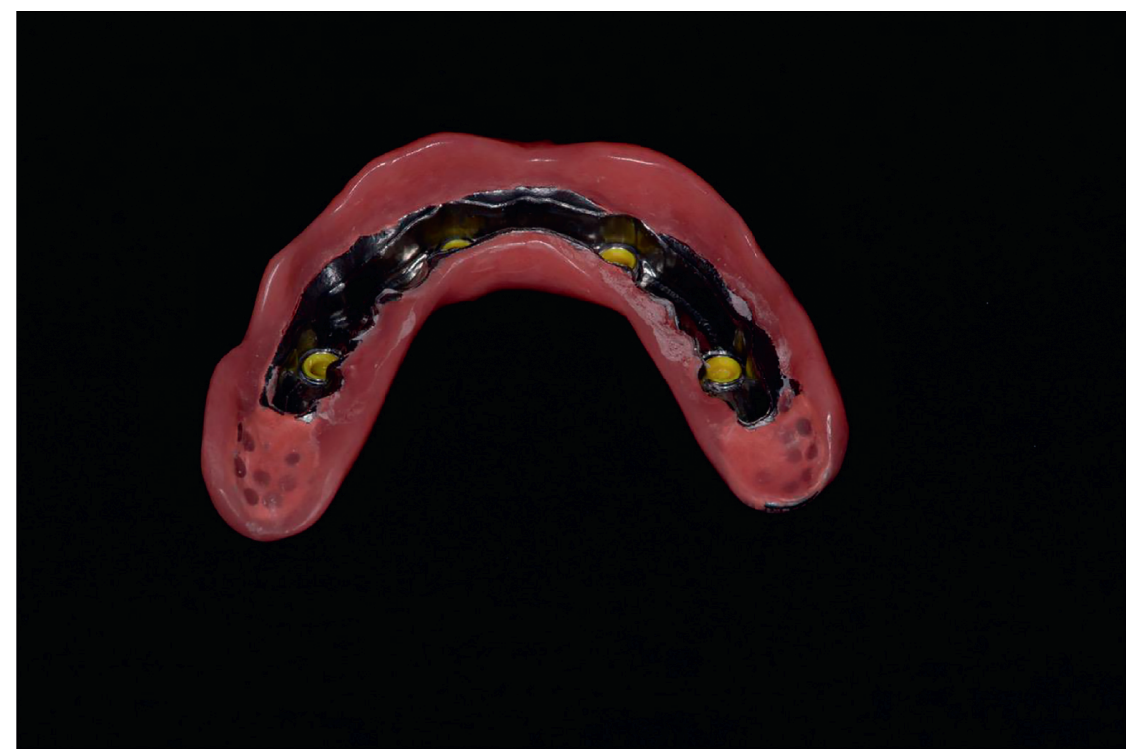

FIgURE 3: View of the internal surface of the overdenture.

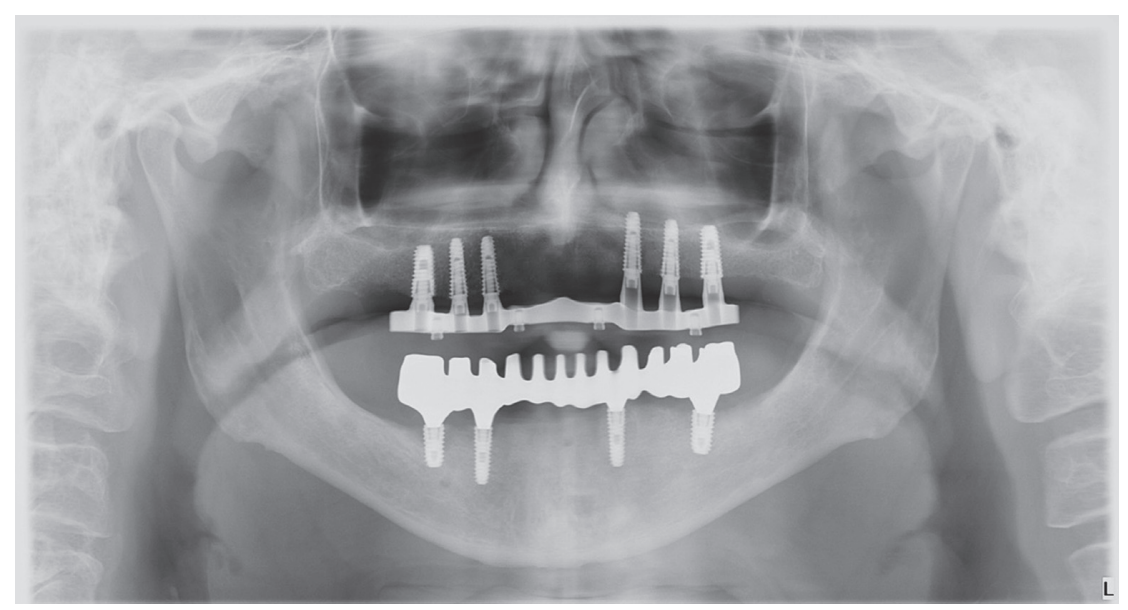

FIgURE 4: Final panoramic radiograph two years after overdenture delivery.

evaluate statistically significant differences between centers for implant and prosthetic failures and complications. A comparison of the means for OHIP scores between the baseline and the follow-ups was performed by paired tests. Patients were grouped based on their facial type assessment (brachycephalic, dolichocephalic, and mesocephalic) and treated arch (mandible and maxilla). The mean differences in MBL and OHIP between different subgroups were compared using a mixed-model repeated-measures analysis of variance (ANOVA).

\section{Results}

A total of 43 patients (14 males and 29 females), with a mean age of 69.7 years, were treated with an implant overdenture supported by a metal bar and low-profile attachments. Fiftyone patients were originally screened for eligibility, but eight were excluded. Two were heavy smokers, the other two needed postextractive implants, and the other four refused a removable option.

Seventeen patients were treated in the mandible (67 implants, range three to four) and 26 in the maxilla (110 implants, range three to six). Nine out of 43 patients were smokers, while 16 patients presented at least one mild systemic disease (American Society of Anesthesiologists Classification, ASA 2). A total of 13 patients were Cawood and Howell class III; 19 patients were class IV; five patients were class $\mathrm{V}$, and the last six patients were class VI.

Overall, 177 implants were placed (range three to six) to support 43 metal bars with 170 OT Equators (Rhein 83, range three to six). Eleven metal bars were fabricated using CAD/CAM technology, while the other 32 were conventionally produced using casting technique. Otherwise, 37 counterparts were produced with conventional casting and only six with laser melting technology. Definitive prostheses had either composite (15) or acrylic resin (28) teeth. Final 
occlusion with anterior guidance was developed in six cases, group function of the working side in 20 cases, and full balanced occlusion in the other 17 cases. Patients were also classified based on their facial type assessment. Four were brachycephalic, three were dolichocephalic, and 36 were mesocephalic.

All the participants were followed up for at least two years (mean follow-up 42.2 months, range 24-88) after prosthesis delivery. At the two-year follow-up examination, two maxillary implants failed in one nonsmoking patient, six months after definitive prosthesis delivery, scoring an implant survival rate of $98.9 \%$. Implants were removed. After 4 months of healing, new implants were placed and a new prosthesis was delivered. The 2-year prosthesis survival rate was $97.7 \%$.

Only three minor technical complications were reported during the follow-up (7\%). Two complications were experienced at the one-year follow-up examination in two patients of different centers, resulting in premature replacement of a retentive cap. The last complication was reported in another center 18 months after the delivery of the final restoration. In this case, a precontact of the prosthesis likely caused the wear of the attachment that was replaced, and the occlusion was checked. No statistically significant difference was found between centers for implant failure $(P=0.2345)$ and complications $(0.1307)$.

One year after implant loading, the mean marginal bone loss of $0.26 \pm 0.16 \mathrm{~mm}$ ( $95 \%$ CI: 0.13 to $0.27 \mathrm{~mm}$ ) was reported. At the second-year follow-up examination, the mean marginal bone loss remained stable $(0.22 \pm 0.09 \mathrm{~mm})(95 \%$ CI: 0.16 to $0.26 \mathrm{~mm})$. The difference was not statistically significant $(P=0.3349)$.

The OHIP score at baseline was $70.7 \pm 6.7$ (95\% CI from 67.4 to 72.6 ). One year after the delivery of the implantretained overdenture, OHIP was $22.3 \pm 5.9$ (95\% CI from 19.5 to 24.5$)$. The difference was statistically significant ( $48.4 \pm 6.1 ; 95 \%$ CI from 46.9 to $51.4 ; P \leq 0.001)$ with a better value at the one-year follow-up examination. Two years after the delivery of the implant-retained overdenture, OHIP was $22.3 \pm 7.1$ (95\% CI from 17.4 to 24.6 ). The difference was statistically significant ( $48.5 \pm 6.8 ; 95 \%$ CI from 44.1 to 52.6 ; $P \leq 0.001)$ with a better value at the one-year follow-up examination.

At the two-year follow-up session, the bleeding index was $0.11 \pm 0.13(0.00 ; 0.00 ; 0.06 ; 0.13 ; 0.50)$, while the plaque index was $0.10 \pm 0.08(0.00 ; 0.03 ; 0.13 ; 0.13 ; 0.25)$. In both cases, difference from the one-year follow-up examination was not statistically significant $(P=0.2882$ and $P=0.4862$, respectively).

Patients were grouped based on their facial type assessment (brachycephalic, dolichocephalic, and mesocephalic) and treated arch (mandible and maxilla). Regarding facial type assessment, all failed implants and complications were experienced in mesocephalic patients. Nevertheless, difference between the facial types was not statistically significant for both failures $(P=1.0)$ and complications $(P=1.0)$. Similarly, all failed implants and complications were experienced in the maxilla. Nevertheless, difference compared to the mandible was not statistically
TABle 2: Distribution of implants failures and complications between subgroups.

\begin{tabular}{lcccc}
\hline & Implants & Prostheses & Failures & Complications \\
\hline Brachycephalic & 16 & 4 & & \\
Dolichocephalic & 12 & 3 & & \\
Mesocephalic & 149 & 36 & 2 & 3 \\
Maxilla & 110 & 26 & 2 & 3 \\
Mandible & 67 & 17 & & \\
\hline
\end{tabular}

significant for both failures $(P=0.5268)$ and complications $(P=0.2658)$ (Table 2). At the two-year follow-up examination, differences in MBL and OHIP between subgroups were not statistically significant in all the subgroups (facial type: OHIP $(P=0.3185)$ and MBL $(P=0.7561)$; treated arch: OHIP $(P=0.5915)$ and MBL $(P=0.6318))$.

\section{Discussion}

The purpose of this study was to present the two-year followup data of implant and prosthetic survival and success rates, patient satisfaction, and peri-implant bone loss of complete edentulous patients treated with a complete maxilla or mandible hybrid fixed/removable overdenture supported by a CAD/CAM or a conventional cast metal bar, and retained with three to six threadable OT Equator attachments (Rhein 83) placed along the implant bar. The successful implant (98.9\%) and prosthetic (97.7\%) survival rates, low complications rate, and good periodontal parameters, as well as a mean bone loss of $0.22 \pm 0.09 \mathrm{~mm}$ ( $95 \%$ CI 0.16 to 0.26 ) experienced at the last follow-up examinations, are consistent with other studies investigating the same topic $[10,11]$.

Patients' and clinician's psychological acceptance of hybrid fixed/removable solutions still remains a major concern. Patients should be informed that a fixed dental prosthesis on four implants may be a possible alternative to an overdenture fully supported by implants. Nevertheless, it is associated with higher marginal bone loss, high frequency of complications, and poor plaque control, particularly in extremely atrophic patients [12,16-18]. Moreover, lip and cheek support were not sufficiently supported using fixed prosthesis compared with a removable overdenture or a complete removable prosthesis [9].

In the present study, a trend of minimum marginal bone loss and good periodontal parameters was observed within the two-year follow-up. A possible explanation could be that a good level of hygiene can be expected using the specific bar design with the low-profile attachments that provides additional retention similar to fixed implant-supported prosthesis. According to the recent literature, patients with a history of periodontitis manifest greater probing depths, more marginal bone loss surrounding implants, and a higher incidence of peri-implantitis [1-3]. Carefully monitored supportive periodontal therapy and proper management are mandatory. For the latter, an implant-supported overdenture with a metal bar and low-profile attachments could be easier to maintain, allowing for longer successful followup $[19,20]$. Moreover, similar to a fixed dental prosthesis, 
patient satisfaction significantly improves owing to an improvement in esthetics and masticatory function. Esthetics is one of the most important parameters from the patient point of view. With a hybrid prosthesis, the lips and cheeks can be supported by the prosthetic flanges [10]. Moreover, a hybrid design with pink esthetics allows for a more natural appearance of the restored missing teeth and tissues.

Because it was designed as a single-cohort, proof-ofconcept study, the main limitations were the lack of a control group and a small sample size. Nevertheless, 177 implants were placed in 43 patients to support the same number of metal bars with 170 OT Equators (Rhein 83). Patients were also grouped based on their facial type assessment (brachycephalic, dolichocephalic, and mesocephalic) and treated arch (mandible and maxilla) failing to found any statistically significant difference. Hence, these preliminary results may be generalized, and the overall investigation should be considered as a pilot for future multicenter randomized clinical trials with control group comparison.

\section{Conclusions}

Implant overdenture supported by a CAD/CAM titanium bar may be a reliable option for the treatment of the edentulous arches over a 2-year period. Oral health-related quality of life significantly improved in all the treated participants. Further studies on this topic could be an innovative issue in this field and could be an open innovative matter of debate in the literature by adding new information.

\section{Data Availability}

In general, clinical cases and results can be found on social networks (https://www.facebook.com/rhein.italia/, https:// www.researchgate.net/profile/Marco_Tallarico) https://www. rhein83.com/scientific-literature-2/.

\section{Conflicts of Interest}

The authors declare that they have no conflicts of interest.

\section{References}

[1] G. Isola, A. Polizzi, S. Santonocito, A. Alibrandi, and S. Ferlito, "Expression of salivary and serum malondialdehyde and lipid profile of patients with periodontitis and coronary heart disease," International Journal of Molecular Sciences, vol. 20, no. 23, Article ID 6061, 2019.

[2] G. Isola, A. Alibrandi, M. Currò et al., "Evaluation of salivary and serum asymmetric dimethylarginine (ADMA) levels in patients with periodontal and cardiovascular disease as subclinical marker of cardiovascular risk," Journal of Periodontology, vol. 91, no. 8, pp. 1076-1084, 2020.

[3] G. Isola, A. Polizzi, V. Iorio-Siciliano, A. Alibrandi, L. Ramaglia, and R. Leonardi, "Effectiveness of a nutraceutical agent in the non-surgical periodontal therapy: a randomized, controlled clinical trial," Clinical Oral Investigations, 2020.

[4] N. Sargolzaie, A. Moeintaghavi, and H. Shojaie, "Comparing the quality of life of patients requesting dental implants before and after implant," The Open Dentistry Journal, vol. 11, no. 1, pp. 485-491, 2017.
[5] D. Harris, S. Höfer, C. A. O’Boyle et al., "A comparison of implant-retained mandibular overdentures and conventional dentures on quality of life in edentulous patients: a randomized, prospective, within-subject controlled clinical trial," Clinical Oral Implants Research, vol. 24, no. 1, pp. 96-103, 2013.

[6] T. Bozini, H. Petridis, K. Garefis, and P. Garefis, "A metaanalysis of prosthodontic complication rates of implantsupported fixed dental prostheses in edentulous patients after an observation period of at least 5 years," The International Journal of Oral \& Maxillofacial Implants, vol. 26, no. 2, pp. 304-318, 2011.

[7] P. Papaspyridakos, C. J. Chen, S. K. Chuang, H. P. Weber, and G. O. Gallucci, "A systematic review of biologic and technical complications with fixed implant rehabilitations for edentulous patients," The International Journal of Oral \& Maxillofacial Implants, vol. 27, no. 1, pp. 102-110, 2012.

[8] N. U. Zitzmann and C. P. Marinello, "Fixed or removable implant-supported restorations in the edentulous maxilla: literature review," Practical Periodontics and Aesthetic Dentistry: PPAD, vol. 12, no. 6, pp. 599-608, 2000.

[9] M. Avrampou, R. Mericske-Stern, M. B. Blatz, and J. Katsoulis, "Virtual implant planning in the edentulous maxilla: criteria for decision making of prosthesis design," Clinical Oral Implants Research, vol. 24, pp. 152-159, 2012.

[10] M. Tallarico, E. Xhanari, B. Kadiu, and R. Scrascia, "Implant rehabilitation of extremely atrophic mandibles (Cawood and Howell Class VI) with a fixed-removable solution supported by four implants: one-year results from a preliminary prospective case series study," Journal of Oral Science \& Rehabilitation, vol. 3, no. 2, pp. 32-40, 2017.

[11] A. Pozzi, M. Tallarico, and P. K. Moy, "Four-implant overdenture fully supported by a CAD-CAM titanium bar: a single-cohort prospective 1-year preliminary study," The Journal of Prosthetic Dentistry, vol. 116, no. 4, pp. 516-523, 2016.

[12] M. Tallarico, L. Canullo, M. Pisano, D. Peñarrocha-Oltra, M. Peñarrocha-Diago, and S. M. Meloni, "An up to 7-year retrospective analysis of biologic and technical complication with the all-on-4 concept," Journal of Oral Implantology, vol. 42, no. 3, pp. 265-271, 2016.

[13] E. Elm von, D. G. Altman, M. Egger et al., "Strengthening the reporting of observational studies in epidemiology (STROBE) statement: guidelines for reporting observational studies," Journal of Clinical Epidemiology, vol. 61, no. 4, pp. 344-349, 2008.

[14] J. I. Cawood and R. A. Howell, "A classification of the edentulous jaws," International Journal of Oral and Maxillofacial Surgery, vol. 17, no. 4, pp. 232-236, 1988.

[15] J. Abduo, V. Bennani, N. Waddell, K. Lyons, and M. Swain, "Assessing the fit of implant fixed prostheses: a critical review," International Journal of Oral \& Maxillofacial Implants, vol. 25, no. 3, pp. 506-515, 2010.

[16] S. B. M. Patzelt, O. Bahat, M. A. Reynolds, and J. R. Strub, "The all-on-four treatment concept: a systematic review," Clinical Implant Dentistry and Related Research, vol. 16, no. 6, pp. 836-855, 2014.

[17] S. J. Sadowsky, "Treatment considerations for maxillary implant overdentures: a systematic review," The Journal of Prosthetic Dentistry, vol. 97, no. 6, pp. 340-348, 2007.

[18] M. Tallarico, S. M. Meloni, L. Canullo, M. Caneva, and G. Polizzi, "Five-year results of a randomized controlled trial comparing patients rehabilitated with immediately loaded maxillary cross-arch fixed dental prosthesis supported by four 
or six implants placed using guided surgery," Clinical Implant Dentistry and Related Research, vol. 18, no. 5, pp. 965-972, 2016.

[19] D. French and M. Tallarico, "Eight-year clinical and radiologic results of maxillary and mandibular implant-retained bar overdentures carried out on oxidized (TiUnite ${ }^{\mathrm{TM}}$ ) replace select implants placed in regenerated bone: a clinical case," Quintessence International, vol. 45, no. 2, pp. 135-140, 2014.

[20] C. A. Babbush and M. Tallarico, "Twelve-year clinical and radiological results of maxillary and mandibular implantretained bar overdentures carried out on oxidized (TiUnite) replace select implants: a clinical case," Journal of Oral Implantology, vol. 39, no. 6, pp. 737-742, 2013. 